\title{
Dynamic resistance measurement in a four-tape YBCO stack with various applied field orientation
}

\author{
Yanchao Liu, Zhenan Jiang, Quan Li, Chris W. Bumby, Rodney A. Badcock, and Jin Fang
}

\begin{abstract}
The dynamic resistance which occurs when a superconductor carrying DC current is exposed to alternating magnetic field plays an important role in HTS applications such as flux pumps and rotating machines. We report experimental results on dynamic resistance in a four-tape coated conductor stack when exposed to AC magnetic fields with different magnetic field angles (the angles between the magnetic field and normal vector component of the tape surface, $\theta$ ) at $77 \mathrm{~K}$. The conductors for the stack are $4 \mathrm{~mm}$-wide SuperPower SC4050 wires. The field angle was varied from $0{ }^{\circ}$ to 120 ${ }^{\circ}$ at a resolution of $15^{\circ}$ to study the field angle dependence of dynamic resistance on field angle as well as wire $I_{c}(B, \theta)$. We also varied the field frequency, the magnetic field amplitude, and the DC current level to study the dependence of dynamic resistance on these parameters. Finally, we compared the measured dynamic resistance results at perpendicular magnetic field with the analytical models for single wires. Our results show that the dynamic resistance of the stack was mainly, but not solely, determined by the perpendicular magnetic component. $I_{\mathrm{c}}(B, \theta)$ influences dynamic resistance in the stack due to tilting of the crystal lattice of the superconductor layer with regard to buffer layers.
\end{abstract}

Index Terms-Dynamic resistance, HTS stack, Angle dependence.

\section{INTRODUCTION}

$\mathbf{H}$ TS coated conductors have attracted increasing interest in many HTS applications such as rotating machines, magnets, flux pumps and Superconducting Energy Storage Systems (SMES) [1]-[7]. One concern for those applications is dynamic resistance in HTS wires which occurs when the HTS wires carry DC transport current under an alternating magnetic field [8]-[9]. Dynamic resistance results in energy loss, which may cause challenges in the cryogenic system and HTS coil windings. In HTS rotating machines, HTS rotor coils carry DC current exposed to harmonic 'ripple' fields [1], [10]-[13]. There is no measureable dynamic resistance in the rotor windings when the amplitude of external 'ripple' field smaller than a threshold

Manuscript receipt on October 30, 2018. This work was supported by New Zealand MBIE Contract No. RTVU1304. (Corresponding author: Zhenan Jiang)

Yanchao Liu and Jin Fang is with School of Electrical Engineering, Beijing Jiaotong University, Beijing 100044, China. (email: jfang@bjtu.edu.cn)

Zhenan Jiang, Chris W. Bumby, and Rodney A. Badcock are with the Robinson Research Institute, Victoria University of Wellington, PO Box 33436, Lower Hutt 5046, New Zealand. (e-mail: zhenan.jiang@vuw.ac.nz). Zhenan Jiang and magnetic field. However, as soon as the amplitude of the 'ripple" field becomes larger than the threshold magnetic field, a measureable dynamic resistance will occur due to the interaction between DC current and traversing flux [14]-[15]. Understanding dynamic resistance characteristics can provide useful information for HTS rotor designers. In HTS flux pumps, dynamic resistance determines the maximum pumped current and can also be utilized for current switches [16]-[17].

Other experimental and numerical reports on dynamic resistance have focused on single coated conductors under applied magnetic field with various field angles [18]-[19]. In our previous work, we measured a four-tape YBCO stack in perpendicular magnetic field, where each conductor composing the stack carries the same current [20]. In reality, in HTS applications, field angle (the angle between the magnetic field and normal vector component of the coated conductor surface, $\theta$ as defined in Fig. 1) may take any value, rather than always being zero (perpendicular magnetic field). However, there has been no reports on the dependence of dynamic resistance in HTS stacks exposed to external magnetic field with various orientations.

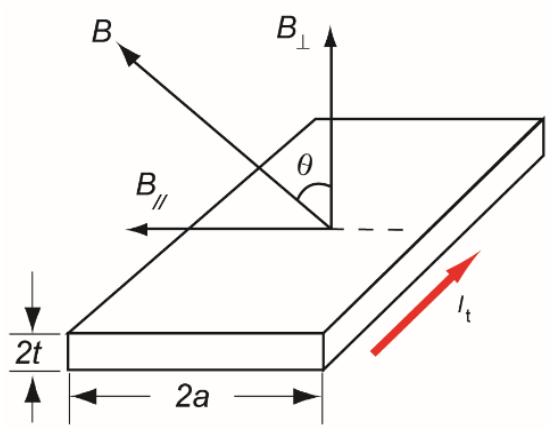

Fig. 1. Definition of field angle, $\theta$

In this work, we present the dependence of dynamic resistance on the applied field angle in a four-tape YBCO stack.

Rodney A. Badcock are also with Beijing Jiaotong University, Beijing 100044, China.

Q. Li is with School of Engineering, University of Edinburgh, Faraday Building. The King's Buildings, Edinburgh EH9 3JL, UK.(e-mail: quan.li@ed.ac.uk)

Color versions of one or more of the figures in this paper are available online at http://ieeexplore.ieee.org.

Digital Object Identifier will be inserted here upon acceptance. 
The field angle was varied from $0{ }^{\circ}$ to $120^{\circ}$ at a resolution of $15^{\circ}$ to investigate the field angle dependence of the dynamic resistance. The DC current level, the magnetic field amplitude, and the frequency of the magnetic field have been varied to study the influence of these parameters on the dynamic resistance. The measured results in perpendicular magnetic field were compared with the analytical models for single wires.

\section{EXPERIMENTAL METHOD}

Fig. 2 shows the schematics of our experimental set up to measure dynamic resistance in the four-tape stack under external magnetic field with various field angles [20]. An AC magnet composed of two vertical racetrack coils was used to produce AC magnetic field amplitudes up to $100 \mathrm{mT}$ [20]. Two resonant frequencies of the magnetic field were chosen by adjusting the value of capacitance of a serially connected capacitor bank in the circuit to explore the frequency dependence of the dynamic resistance of the stack. A CROWN K2 audio amplifier was used to energize the magnet. The HTS stack was mounted on the top of the machined surface of a GFRP sample holder and then placed in the center of the magnet. The field angle, $\theta$, was varied by rotating the sample holder inside the magnet. In this work, a 4 mm-wide SCS4050 YBCO wire with self-field $I_{\mathrm{c}}$ of $104 \mathrm{~A}$ manufactured by SuperPower Inc. was used to assemble the four-tape YBCO stack sample. The specifications of the wire are shown in Table 1. Kapton sheets and tapes were used as insulation between the wires composing the stack, and the vertical distance between the neighboring superconductor layers is $\sim 0.295 \mathrm{~mm}$. Each tape carries the same DC current by connecting the tapes in series. Hewlett Packard 6682A DC power supply was used to drive the DC sample circuit. Spiral loops attached on each conductor as shown in Fig. 2 were used to measure voltage in each conductor due to dynamic resistance [21]. The effective distance between the voltage taps is $80 \mathrm{~mm}$. The $\mathrm{DC}$ voltage output from the voltage taps was measured using a dc Hewlett Packard 34420A nano-volt meter.

All measurements were carried out at $77 \mathrm{~K}$. Both the magnet and the stack sample were immersed in the liquid nitrogen.

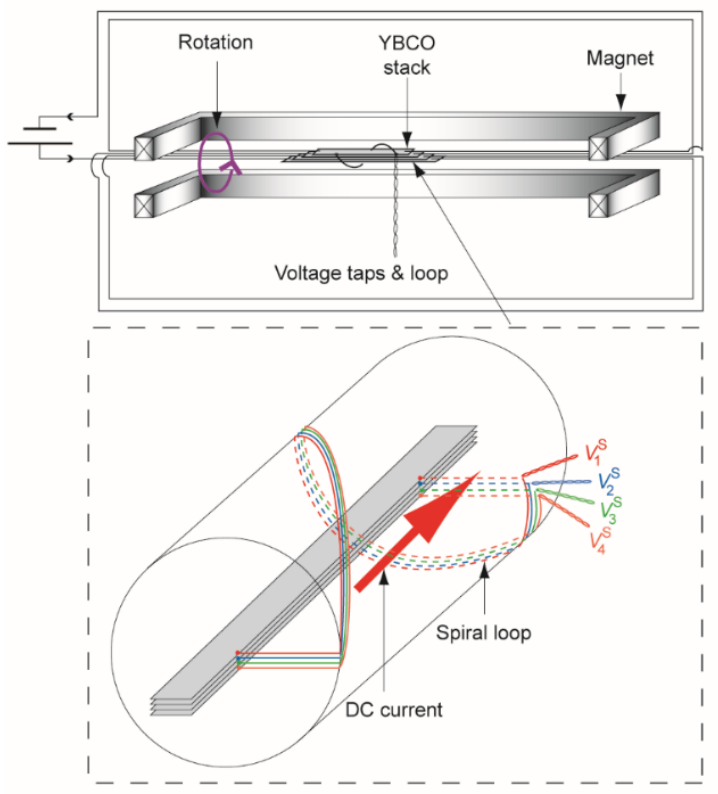

Fig. 2. Experimental set-up for stack dynamic resistance measurement and voltage tap arrangement of for the stack

TABLE 1. SPECIFICATIONS OF THE WIRE FOR STACK SAMPLE

\begin{tabular}{ll}
\hline \hline Manufacture & SuperPower (SCS44050) \\
Self-field critical current (A) & 104.0 \\
Sample width $(\mathrm{mm})$ & 4.0 \\
Thickness of Cu stabilizer layer each side $(\mu \mathrm{m})$ & 20 \\
Thickness of superconductor layer $(\mu \mathrm{m})$ & 1.0 \\
Thickness of substrate $(\mu \mathrm{m})$ & 50.0 \\
Distance between neighboring superconductor layers $(\mu \mathrm{m})$ & 295.0 \\
\hline \hline
\end{tabular}

\section{EXPERIMENTAL RESULTS}

\section{A. $I_{c}$ measurement of the wires before and after assembling} the stack

The $I_{\mathrm{c}}$ values of the four wires before and after assembling the stack are shown in Table 2. The $I_{\mathrm{c}}$ values of the wires became smaller in the stack environment than self-field $I_{\mathrm{c}}$ values of the single wires due to the superposition of the magnetic fields generated by other tapes [20].

The $I_{\mathrm{c}}$ values of the two outer tapes (T1 and T4) are smaller than those of the two inner tapes (T2 and T3) under stack environment due to the outer tapes experiencing larger magnetic fields than the inner tapes [22].

TABEL 2. $I_{\mathrm{c}}$ VALUE Of WiRe Before AND AFter AsSEMBling

\begin{tabular}{llllll}
\hline \hline & $\mathrm{T} 1$ & $\mathrm{~T} 2$ & $\mathrm{~T} 3$ & $\mathrm{~T} 4$ & Average \\
\hline Before assembling (A) & 105.7 & 105.3 & 104.6 & 104.6 & 105.1 \\
After assembling (A) & 81.6 & 85.4 & 85.3 & 83.9 & 84 \\
\hline \hline
\end{tabular}

\section{B. $R_{d y n}$ values in the stack under perpendicular magnetic field}

The dynamic resistance for a single coated conductor shown in Fig. 1 under perpendicular magnetic field per unit length per cycle, $R_{\mathrm{dyn}}$, can be calculated by [23]-[24],

$$
\frac{R_{d y n}}{f l}=\frac{4 a}{I_{c 0}}\left(B_{a}-B_{t h}\right)
$$


where, $I_{\mathrm{c} 0}$ is the self-field critical current of the conductor, $a$ is the half-width of the conductor, $l$ is the distance between the two voltage taps, $B_{\mathrm{a}}$ is the amplitude of the applied magnetic field, and $f$ is the frequency of the applied magnetic field. $B_{\mathrm{th}}$ is the threshold magnetic field, which is given by,

$$
B_{t h}=B_{e p}(1-i)=B_{e p}\left(1-\frac{I_{t}}{I_{c 0}}\right)
$$

where $i$ is $I_{\mathrm{t}} / I_{\mathrm{c} 0}$ and $\left(1-I_{\mathrm{t}} / I_{\mathrm{c} 0}\right)$ is the filling portion of the DC current and $B_{\mathrm{ep}}$ is the effective penetration field [25]-[26]. In our previous work, we have derived an analytical equation for the calculation of the $B_{\text {th }}$ value in coated conductors exposed to perpendicular magnetic fields. We have shown that $B_{\text {ep }}=$ $2.4642 \mu_{0} t J_{\mathrm{c} 0} / \pi$ [23]. An alternative equation of $B_{\mathrm{th}}$ was derived by Mikitik and Brandt, $B_{\mathrm{th}, \mathrm{MB}}$ [24],

$B_{t h, M B}=\frac{\mu_{0} J_{c 0} t}{2 \pi}\left[\frac{1}{i} \ln \left(\frac{1+i}{1-i}\right)+\ln \left(\frac{1-i^{2}}{4 i^{2}}\right)\right]$

Previous work has shown that for a single coated conductor, Eq. (2) provides a better agreement with the experimentally derived $B_{\text {th }}$ values for $i \geq 0.2$ than the values from Eq. (3). However, for $i<0.1, B_{\text {th }}$ values from Eq. (3) have better agreement with the experimentally derived $B_{\text {th }}$ [23].

We measured dynamic resistance values of each tape in the stack at $67.89 \mathrm{~Hz}$ and $87.65 \mathrm{~Hz}$ for different DC current levels under perpendicular $\mathrm{AC}$ magnetic fields to investigate the frequency dependency of dynamic resistance in the stack. The measured dynamic resistance values at the two frequencies agreed with each other, and this implies the hysteretic nature of dynamic resistance in the stack [20].

Fig. 3 shows the measured $R_{\text {dyn }}$ in each tape for 5 different current values at $67.89 \mathrm{~Hz}$ in perpendicular field $\left(0^{\circ}\right)$. The $R_{\text {dyn }}$ are normalized by the sample length and magnetic field frequency, and are plotted as a function of the amplitude of the applied magnetic field. The theoretical values in a single coated conductor obtained from Eqns. (1) and (2) are also shown in the figure. The result shown in Fig. 3 are similar to the results in our 4-tape stack even though the wire $I_{\mathrm{c}}$ values used in the stacks in this work are different [20]. In the following we recap the main characteristics of the result in the stack:

1) The $R_{\mathrm{dyn}}$ values in each conductor at each current level broadly agree with one another.

2) The $B_{\text {th }}$ values for the stack (which are extracted from the $x$ - axis intercept of linear fits of the measured data) decrease with increasing DC current levels.

3) The $B_{\text {th }}$ values for the stack are bigger than those in the single conductor at each DC current. This is due to the shielding effect arising from circulating currents in each conductor in the stack.

4) The gradient of the linear fits $\left(\mathrm{d} R_{\mathrm{dyn}} / \mathrm{d} B_{\mathrm{a}}\right)$ for each tape in the stack are much larger than the single conductor.
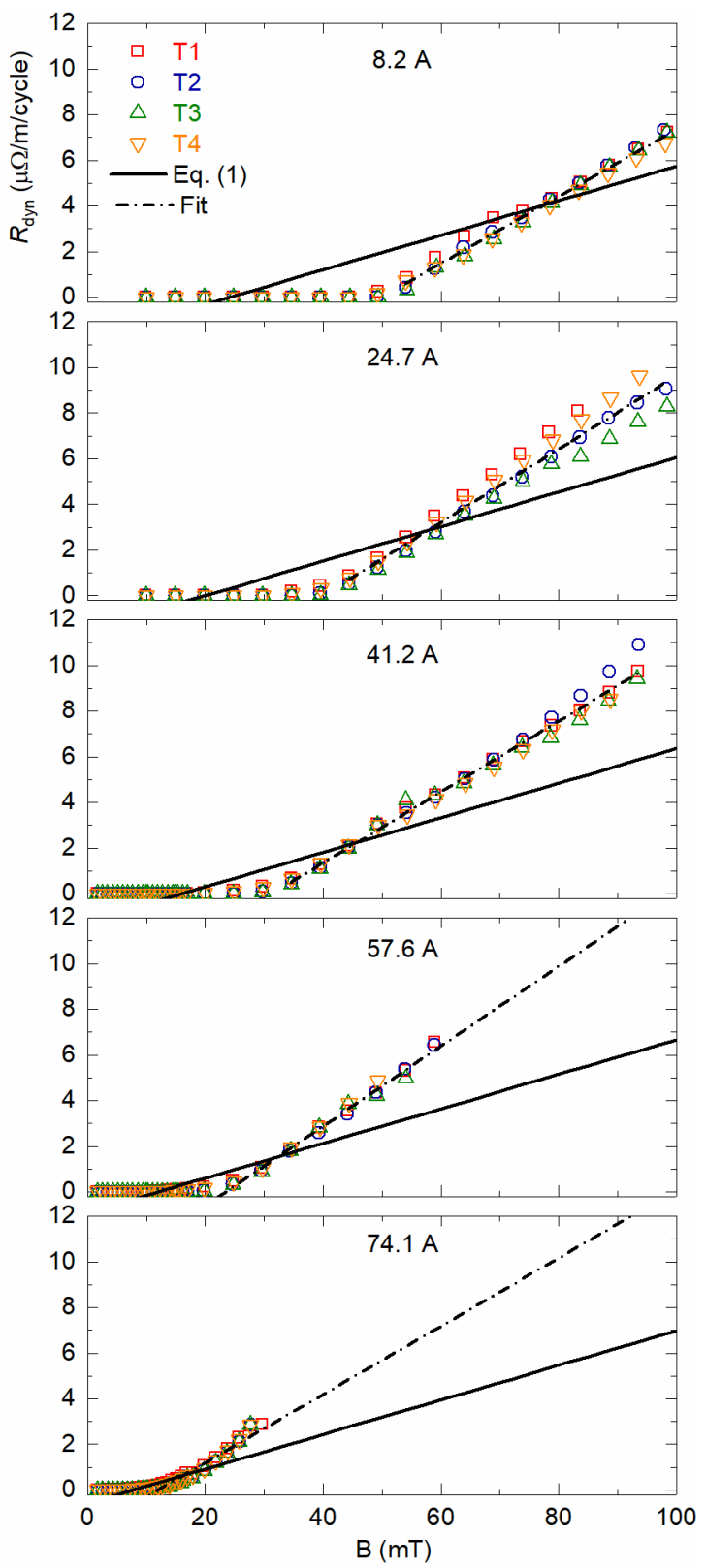

Fig. 3. Measured dynamic resistance in each wire of the YBCO stack at 5 different current for $67.89 \mathrm{~Hz}$ under perpendicular magnetic field compared with the calculated results in the single tape by Eq. (1).

Fig. 4 shows the comparison of the experimentally obtained $B_{\text {th }}$ values for the stack (one is from T1 and the other is the averaged value of all four tapes), measured and analytical $B_{\text {th }}$ values for the single conductor in perpendicular magnetic field at various different $\mathrm{DC}$ current values ranging from $1 \mathrm{~A}$ to $100 \mathrm{~A}$. The analytical $B_{\text {th }}$ values are from Eqs. (2) and (3). It is worth noting that the $B_{\text {th }}$ values for the stack from T1 and from the averaged value of the $B_{\text {th }}$ values in all four tapes agree from one and another. As we observed in [20] before, the $B_{\text {th }}$ values of the stack are bigger than those for the single conductor in wide range of current values $(8.2 \mathrm{~A}-74.1 \mathrm{~A})$ due to the shielding effect. The difference between the stack and the single wire increases with decreasing the DC current value because the shielding effect is more significant at small transport current. 
When $I_{\mathrm{t}}$ is bigger than $8.2 \mathrm{~A}$, the $B_{\mathrm{th}}$ of stack approximately decreases linearly with increasing the DC current. The trend is similar to single tape in the same DC current range. However, interestingly, when $I_{\mathrm{t}}<8.2 \mathrm{~A}$, the $B_{\text {th }}$ values for the stack approach the analytical values from Eq. (3). For a single coated conductor, when $I_{\mathrm{t}}$ is very small, the $B_{\text {ep }}$ value increases due to the increase in the shielding currents [27]. As a result, $B_{\text {th }}$ values for the single conductor show good agreement with Eq. (3) at very small $I_{\mathrm{t}}$. Same thing might happen in the conductors in the stack [27].

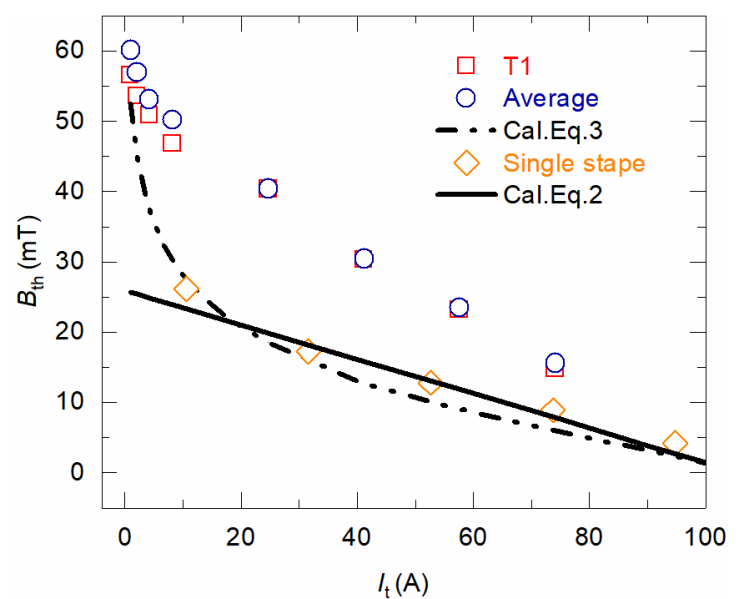

Fig. 4. Comparison of the $B_{\text {th }}$ values of the stack and the single tape.
C. Angle dependence of the dynamic resistance
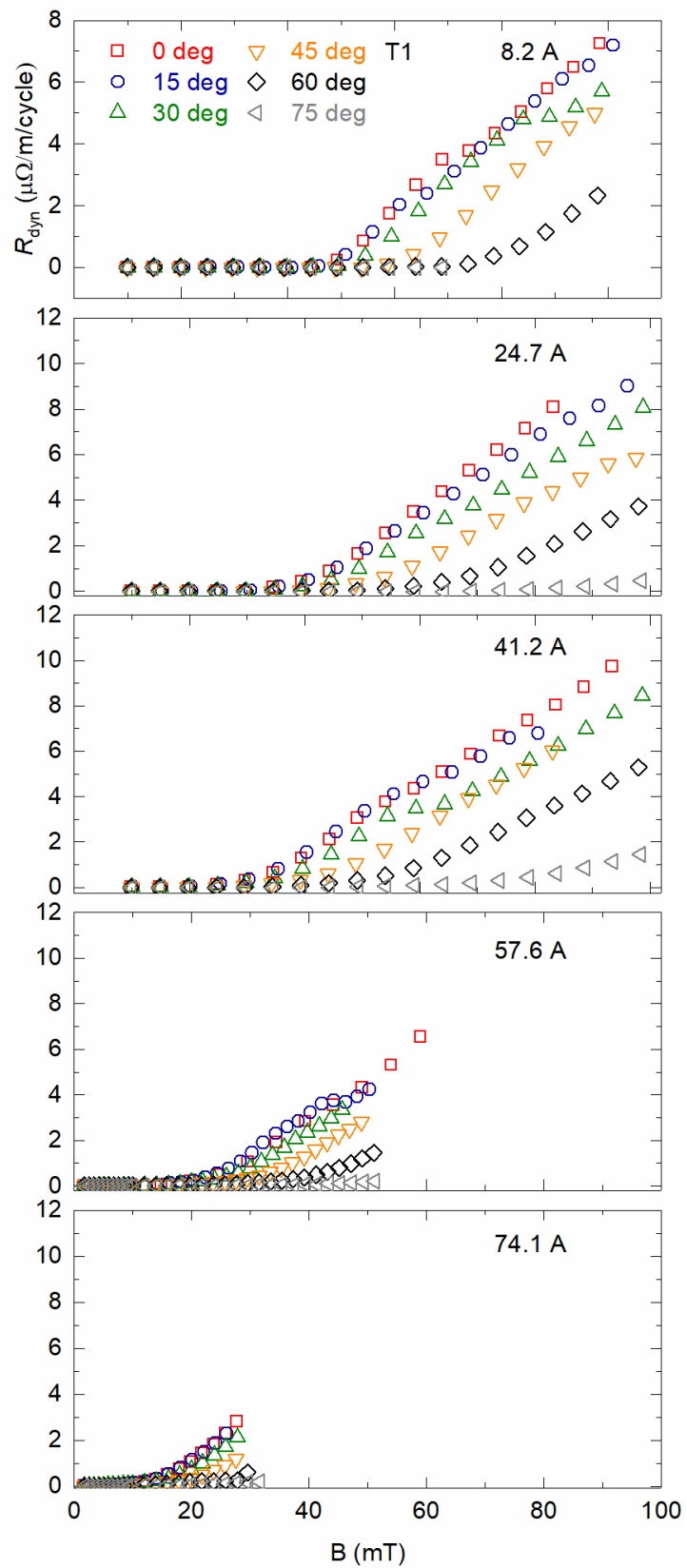

Fig. 5. Measured $R_{\mathrm{dyn}}$ values of $\mathrm{T} 1$ at various field angles plotted as a function of the applied magnetic field amplitude at 5 different currents at $67.89 \mathrm{~Hz}$.

Fig. 5 shows the measured $R_{\text {dyn }}$ values obtained from $0^{\circ}$ to $75^{\circ}$ at a resolution of $15^{\circ}$ for 5 applied current values plotted as a function of the magnetic field amplitude. The results for all the tapes are similar in all cases, therefore we only show T1 result here. The results in the stack are similar to the results observed in our previous study for single wires, i.e. $\mathrm{d} R_{\mathrm{dyn}} / \mathrm{d} B_{\mathrm{a}}$ becomes larger with decreasing $\theta$ and the $B_{\text {th }}$ values decrease with decreasing $\theta$ [27]. No measurable values of $R_{\text {dyn }}$ under parallel magnetic field $\left(\theta=90^{\circ}\right)$ at any of the transport currents were observed in this work.

In [27], it was concluded that the $R_{\text {dyn }}$ of single coated conductor are mainly determined by the perpendicular component of the applied magnetic field, and the parallel magnetic field plays almost no role in the dynamic resistance of a coated conductor - due to the large aspect ratio (a/t) compared with the 
BSCCO wires. In order to check the effect of the perpendicular component of the applied magnetic field on the $R_{\mathrm{dyn}}$ of the YBCO stack, we have replotted the data as a function of the perpendicular component of the applied magnetic field, $B_{\mathrm{a}} \cdot \cos \theta$, as shown in Fig. 6 .
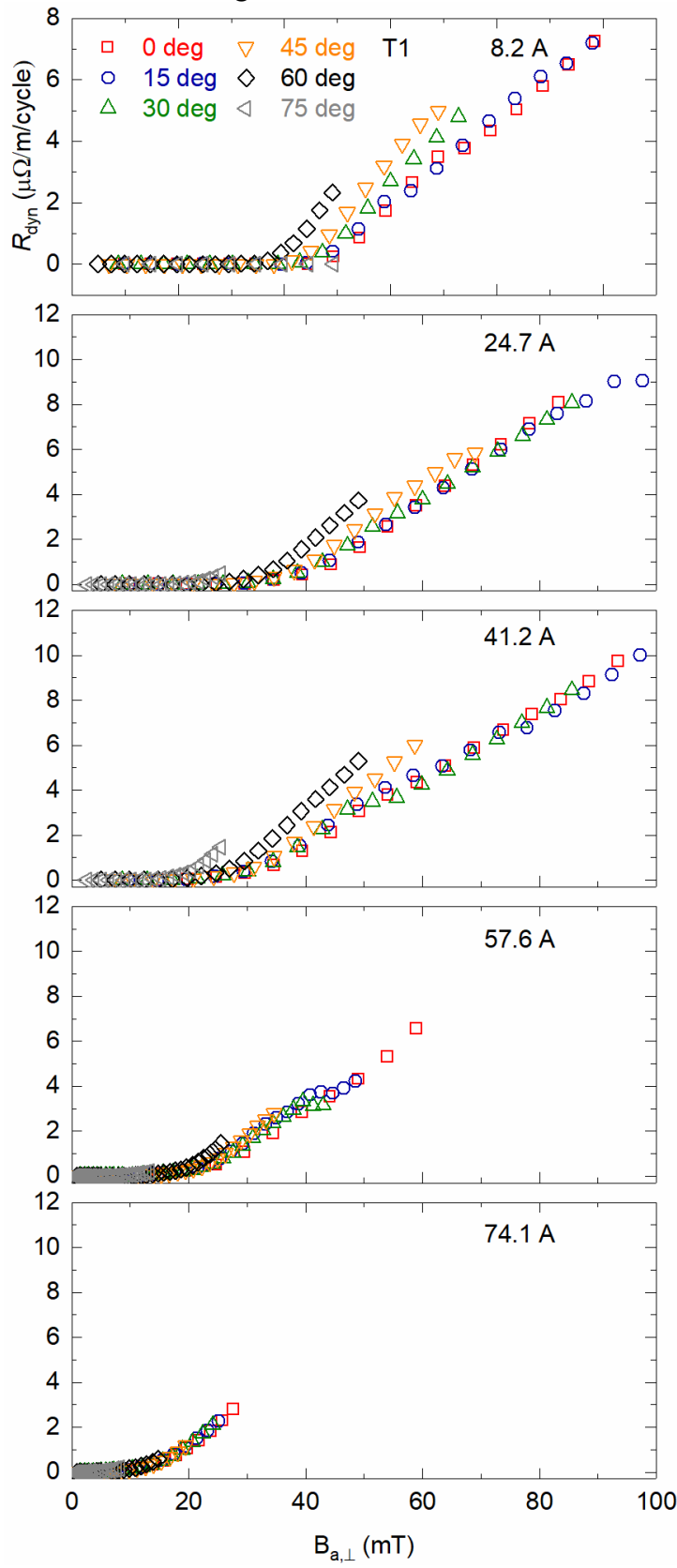

Fig. 6. Measured $R_{\mathrm{dyn}}$ values of $\mathrm{T} 1$ at various field angles plotted as a function of perpendicular magnetic field component $B_{\mathrm{a}}{ }^{*} \cos \theta$. Data obtained at 5 different currents at $67.89 \mathrm{~Hz}$.

As shown in the figure, the $R_{\mathrm{dyn}}$ values for $\theta=0^{\circ}, 15^{\circ}$, and $30^{\circ}$ broadly agree with each other for all the current values. However, the results for larger field angles deviate from the main curve. The agreement between the results for different field angles improves with increasing the DC current values. However it is clear that -in contrast to the single wire case [27] - a simple perpendicular field dependence is not sufficient to describe the angular dependence of the stack. The difference in the field angle dependence of the dynamic resistance might be due to the additive effect of off-angle field contributions from each set of planar shielding currents flowing in the other tapes in the stack. This is a complex effect which will require more advanced modelling to fully elucidate. Additional differences with the single tape case include the relatively smaller aspect ratio of the stack than that of a single coated conductor, and the potential for imperfect planar alignment of each conductor in the stack.

\section{Dynamic resistance characteristics under perpendicular magnetic field with low field amplitude}

In Fig. $7, R_{\text {dyn }}$ values under perpendicular magnetic field at 57.6 A and 74.1 A are plotted as a function of magnetic field amplitude. The magnetic field amplitude is up to $18 \mathrm{mT}$ and there was no measureable $R_{\mathrm{dyn}}$ when the current values were 8.2 A, 24.7 A, and 41.2 A. As shown in the figure, the $R_{\text {dyn }}$ values of the two outer tapes (T1 and T4) are larger than those of the two inner tapes (T2 and T3), while the $B_{\mathrm{th}}$ values of the outer tapes are smaller than those of the inner tapes. Similar phenomenon were observed at all other angles. Qualitatively this can be explained using Eqs. (1) and (2) by replacing $I_{\mathrm{c} 0}$ in the equations with the $I_{\mathrm{c}}$ value of each conductor in the stack arrangement. The $I_{\mathrm{c}}$ values of the outer tapes are smaller than those in the inner tapes of the stack as shown in Table 2. Therefore, at the same DC current value, the $i$ values for the outer tapes is larger than those of the inner tapes, and this leads to smaller $B_{\text {th }}$ values and larger $R_{\text {dyn }}$ values. The phenomenon disappears at high magnetic field amplitude. This is because the difference in $I_{\mathrm{c}}$ values of all the tapes becomes smaller at high magnetic field amplitudes.

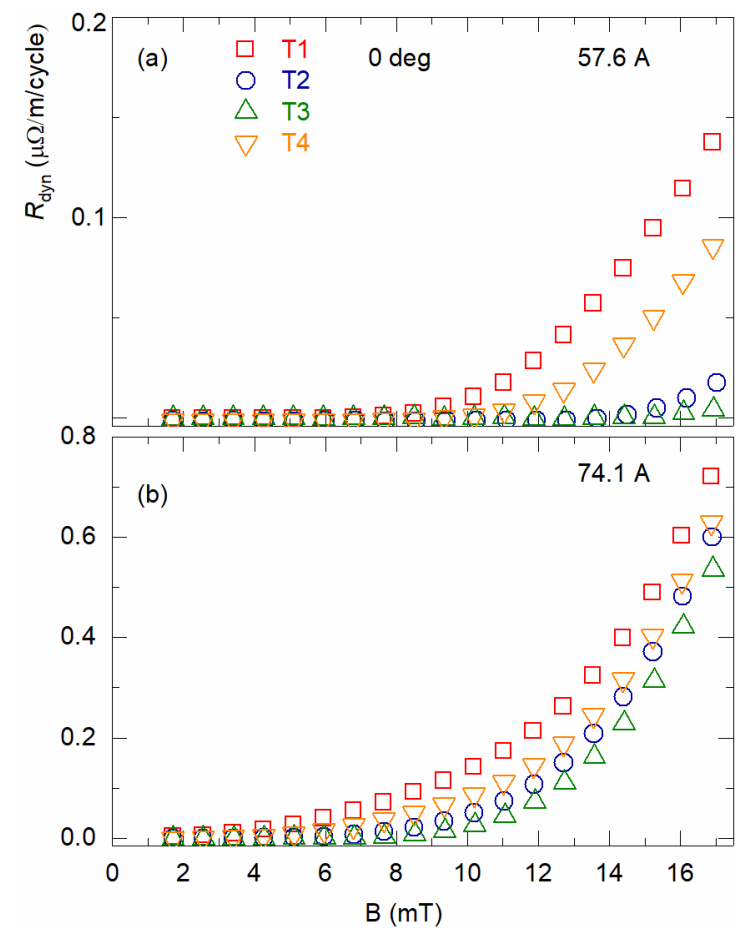

Fig. 7. Measured $R_{\mathrm{dyn}}$ values in each wire under perpendicular magnetic field with the magnetic field amplitude up to $18 \mathrm{mT}$ at $57.6 \mathrm{~A}$ and $74.1 \mathrm{~A}$. 
E. Influence of $I_{c}(B, \theta)$ of sample wires on dynamic resistance

Fig. 8 shows the measured $R_{\text {dyn }}$ values of T1 at $\theta=60^{\circ}$ and $120^{\circ}$ at $5 \mathrm{DC}$ current values plotted as a function of the amplitude of the applied magnetic field. It is obvious that the measured $R_{\mathrm{dyn}}$ value at $60^{\circ}$ is larger than $120^{\circ}$ at all current values. We attribute the result to the anisotropy in the angle dependence of $I_{\mathrm{c}}$ of the coated conductor wires used in the stack [28]. Fig. 9 shows the measured $I_{\mathrm{c}}$ values of a coated conductor, which is cut from the same source material as the conductors used in the stack in this work, at different field angles at $77 \mathrm{~K}$ [19]. The $I_{\mathrm{c}}$ values for $\theta=60^{\circ}$ and $120^{\circ}$ are different as shown in Fig. 9 which is impossible for an isotropic coated conductor. It is immediately apparent that $R_{\mathrm{dyn}}$ values will be different with different $I_{\mathrm{c}}$ values according to Eqs. (1) and (2).

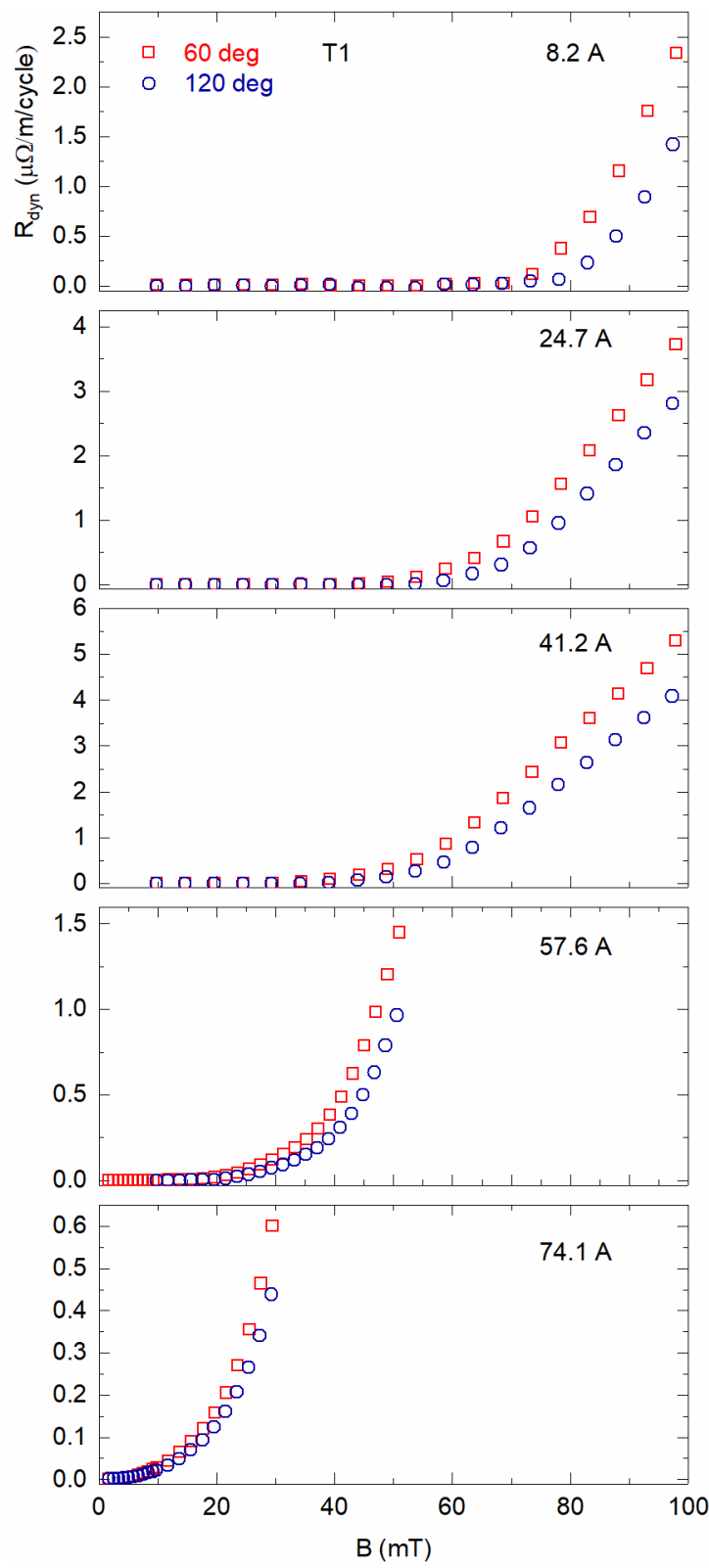

Fig. 8. Comparison of the $R_{\mathrm{dyn}}$ values in $\mathrm{T} 1$ at $60^{\circ}$ and $120^{\circ}$ for $67.89 \mathrm{~Hz}$ under 5 different current values.

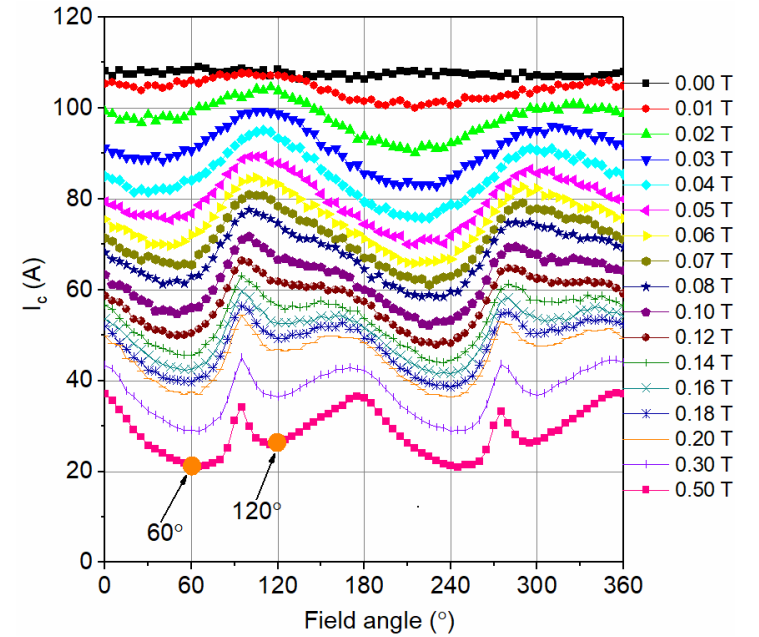

Fig. 9. Measured $I_{\mathrm{c}}(B, \theta)$ values for a single coated conductor at $77 \mathrm{~K}[19]$.
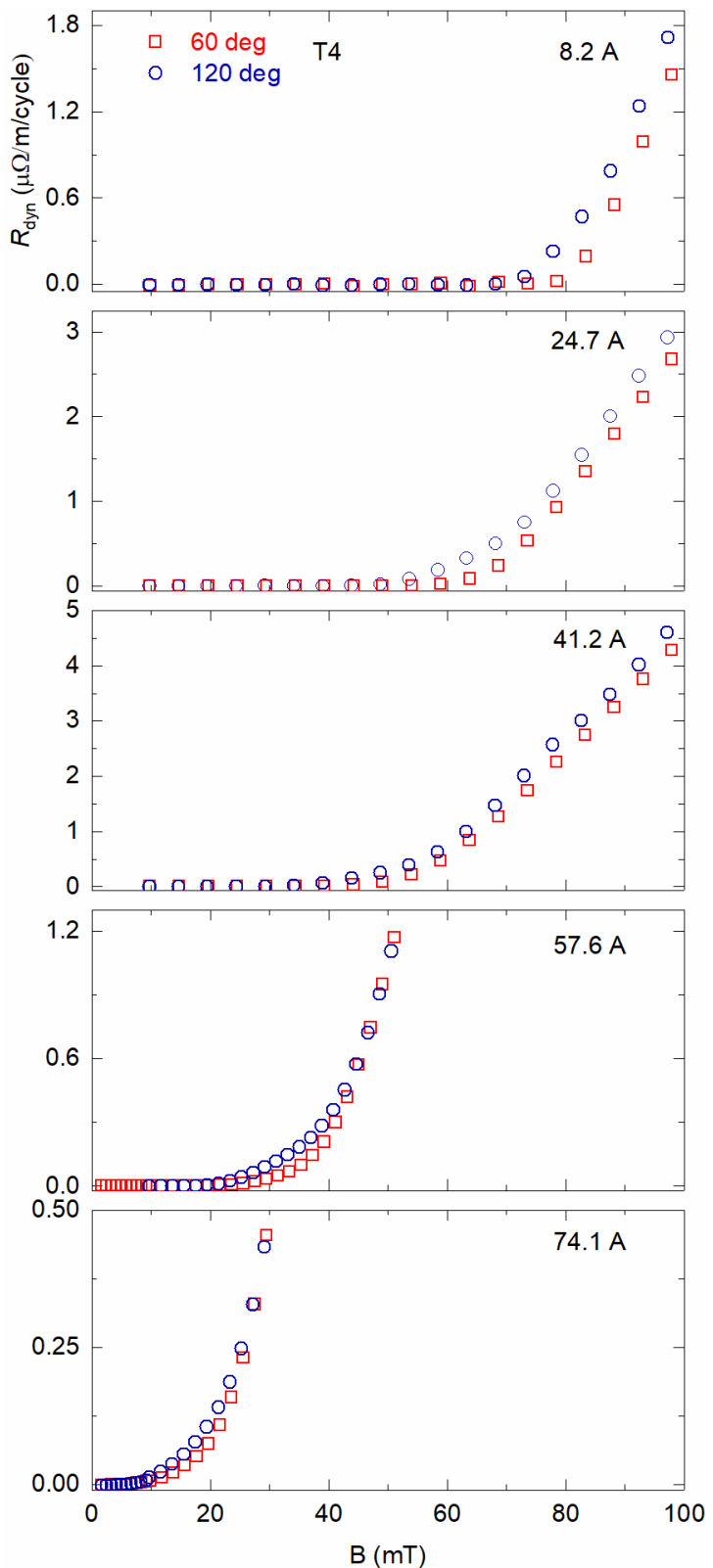

Fig. 10. Comparison of the $R_{\mathrm{dyn}}$ values in $\mathrm{T} 4$ at $60^{\circ}$ and $120^{\circ}$ for $67.89 \mathrm{~Hz}$ under 5 different current values. 
Fig. 10 shows the measured $R_{\mathrm{dyn}}$ values in T4 for $\theta=60^{\circ}$ and $120^{\circ}$. Interestingly, the $R_{\text {dyn }}$ values for $\theta=60^{\circ}$ are smaller than those for $\theta=120^{\circ}$ which is opposite to the results in the other three wires. We attribute the result to possible change of direction of T4 as compared to the other three wires during the sample preparation, i.e. T4 might have been accidentally rotated $180^{\circ}$ in the same sample plane. The crystal lattice of YBCO superconductor layers is normally tilted from the substrate interface, and this results in asymmetry of the $I_{\mathrm{c}}(B, \theta)$ dependence [29]. If we consider a coated conductor exposed to the external magnetic field shown in Fig. 11 (a) which is parallel to the orientation of crystal lattice of the superconductor layer. If the rotation mentioned above happens, the relative angle change between the two cases is $\beta$, as shown in Fig. 11 (b). Therefore, field angle values for the two cases (T1, T2, T3 vs T4) should be different. This gives an explanation in the result from $\mathrm{T} 4$. Similar results have been observed for $\theta=75^{\circ}$ and $105^{\circ}$.

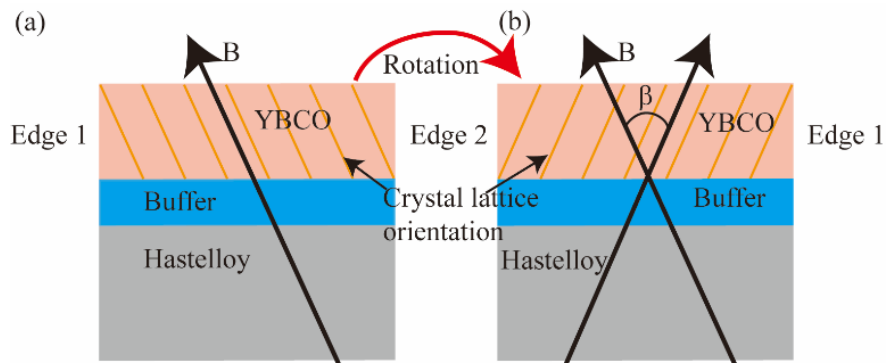

Fig. 11. Schematics of the change of relative angle between the external magnetic field and the orientation of crystal lattice in T4 due to rotation of the tape in the same plane: (a) Zero relative angle before rotation, (b) relative angle $\beta$ after rotation.

\section{CONCLUSION}

We have studied the dependence of dynamic resistance on the field angle of a four-tape YBCO stack while carrying DC currents exposed to AC magnetic fields with various field angles.

Shielding effect was reconfirmed through the difference in $B_{\text {th }}$ values between the stack and a single coated conductor in this work.

At very small DC currents, i.e. when the DC current is smaller than $8.2 \mathrm{~A}$, the $B_{\text {th }}$ value for the stack agrees with theoretical value from Mikitik and Brandt.

Unlike single coated conductors, $R_{\text {dyn }}$ values at different field angles are mainly but not solely determined by perpendicular magnetic field component possibly due to the difference in aspect ratio of the width over thickness of the stack and the single wire.

At $I_{\mathrm{t}}=57.6 \mathrm{~A}$ and $74.1 \mathrm{~A}$, we observed difference in $R_{\mathrm{dyn}}$ values for the outer two tapes and inner two tapes at low magnetic field amplitudes. This should be due to difference in $I_{\mathrm{c}}$ values in the outer and inner tapes when the tapes carry DC current.

$I_{\mathrm{c}}(B, \theta)$ influences dynamic resistance in the stack. This is caused by tilting of the crystal lattice of the superconductor layer with regard to the substrate layers.

\section{ACKNOWLEDGMENT}

The authors thank Mr. Gennady Sidorov for his assistance in the experiment, and Dr. Stuart Wimbush for the $I_{\mathrm{c}}(B, \theta)$ data at $77 \mathrm{~K}$.

\section{REFERENCES}

[1] S. S. Kalsi, K. Weeber, H. Takesue, C. Lewis, H.W. Neumueller, and R. D. Blaugher, "Development Status of Rotating Machines Employing Superconducting Field Windings," Proc. IEEE, vol. 92, no. 10, pp. 16881704, Oct. 2004.

[2] P. J. Masson, D. S. Soban, E. Upton, J. E. Pienkos, and C. A. Luongo, "HTS motors in aircraft propulsion: design considerations," IEEE Trans. Appl. Supercond. vol. 15, pp. 2218 - 2221, 2005.

[3] E. Pardo, "Modeling of AC loss in coils made of thin tapes under DC bias current," IEEE Trans. Appl. Supercond. vol. 24, 2013, Art. no. 4700105.

[4] Z. Jiang, K. Hamilton, N. Amemiya, R. A. Badcock, and C. W. Bumby, “ Dynamic resistance of a high-Tc superconducting flux pump," Appl. Phys. Lett, vol. 105, Sep. 2014, Art. no. 112601.

[5] C. W. Bumby, R. A. Badcock, H. J. Sung, K. M. Kim, Z. Jiang, A. E. Pantoja, P. Bernado, M. Park, and R. G. Buckley, "Development of a brushless HTS exciter for a 10 kW HTS synchronous generator", Supercond. Sci. Technol. vol. 29, 2016, Art. no. 024008.

[6] Z. Jiang, C. W. Bumby, R. A. Badcock, H. J. Sung, N. J. Long, and N. Amemiya, "Impact of flux gap upon dynamic resistance of a rotating HTS flux pump," Supercond. Sci. Technol., vol. 28, Sep. 2015, Art. no. 115008.

[7] M. Strasik, P. E. Johnson, A. C. Day "Design, Fabrication, and Test of a 5-kWh/100-kW Flywheel Energy Storage Utilizing a High-Temperature Superconducting Bearing," IEEE Trans. Appl. Supercond., vol. 17, no. 2, pp. 2133-2137, Jun. 2007.

[8] T. Ogasawara, Y. Takahashi, K. Kanbara, Y. Kubota, K. Yasohama, and K. Yasukochi, "Alternating field losses in superconducting wires carrying dc transport currents: Part 1 single core conductors," Cryogenics, vol. 19, no. 12 , pp. 736-740, Dec. 1979.

[9] M. P. Oomen, J. Rieger, M. Leghissa, B. ten Haken, and H. H. J. ten Kate, "Dynamic resistance in a slab-like superconductor with Jc (B) dependence," Supercond. Sci. Technol., vol. 12, pp. 382-387, 1999.

[10] J. Ogawa, Y. Zushi, M. Fukushima, O. Tsukamoto, E. Suzuki, M. Hirakawa, and K. Kikukawa, "AC losses in a HTS coil carrying DC current in AC external magnetic field", Physica C, vol. 392-396, pp. 1145$1149,2003$.

[11] P. N. Barnes, M. D. Sumption, and G. L. Rhoads, "Review of high power density superconducting generators: Present state and prospects for incorporating YBCO windings," Cryogenics, vol. 45, pp. 670-686, 2005.

[12] V. Lahtinen, E. Pardo, J. Souc, M. Solovyov, and A. Stenvall, "Ripple field losses in direct current biased superconductors: Simulations and comparison with measurements," J. Appl. Phys. vol. 115, Mar. 2014, Art. no. 113907.

[13] V. Lahtinen and A. Stenvall, "The difficulty of modelling ripple field losses in superconductors using the eddy current model," IEEE Trans. Appl. Supercond., vol. 23, no. 3, Jun. 2013, Art. no. 4900505.

[14] R. C. Duckworth, Y. F. Zhang, T. Ha, and M. J. Gouge "Dynamic resistance of YBCO-coated conductors in applied AC fields with DC transport currents and DC background fields," IEEE Trans. Appl. Supercond., vol. 21, pp. 3251-3256, June 2011.

[15] M. Ciszek, O. Tsukamoto, J. Ogawa, and D. Miyagi, "Energy losses in YBCO-123 coated conductors carrying transport current in perpendicular external magnetic field", AIP Conf. Proc. vol. 614, pp. 606-613, 2002.

[16] J. Geng, K. Matsuda, B. Shen, H. Zhang, X. Zhang, L. Fu, Z. Huang, and T. A. Coombs, "HTS Persistent Current Switch Controlled by AC Magnetic Field," IEEE Trans. Appl. Supercond. vol. 26, Apr. 2016, Art. no. 6603306.

[17] J. Geng, and T. A. Coombs, "Mechanism of a high-Tc superconducting flux pump: Using alternating magnetic field to trigger flux flow," Appl. Phys. Lett, vol. 107, Oct. 2015, Art. no. 142601.

[18] M. Ciszek, H. G. Knoopers, J. J. Rabbers, B. t. Haken and H. Kate "Angular dependence of the dynamic resistance and its relation to the $\mathrm{AC}$ transport current loss in Bi-2223/Ag tape superconductors" Supercond. Sci. Technol., vol. 15, pp. 1275-1279, 2002. 
[19] M. D. Ainslie1, C. W. Bumby, Z. Jiang, R. Toyomoto, and N. Amemiya "Numerical modelling of dynamic resistance in high-temperature superconducting coated-conductor wires," Supercond. Sci. Technol., vol. 31, Jun. 2018, Art. no. 074003.

[20] Z. Jiang, W. Zhou, C. W. Bumby, M. Staines, Q. Li, R. A. Badcock, N. J. Long, and, J. Fang "Dynamic resistance measurement of a four-tape YBCO stack in a perpendicular magnetic field," IEEE Trans. Appl. Supercond. vol. 28, Dec. 2018, Art. no. 8200305.

[21] S. Fukui, Y. Kitoh, T. Numata, O. Tsukamoto, J. Fujikami, and K. Hayashi, "Transport current AC losses of high-Tc superconducting tapes exposed to AC magnetic field," Advances in Cryogenic Engineering, vol. 44, pp. 723-730, 1998.

[22] K. Thakur, Z Jiang, M. Staines, N. Long, R. Badcock, and A. Raj "Current carrying capability of HTS Roebel cable" Phys. C, vol. 471, pp. 42-47, 2011.

[23] Z. Jiang, R. Toyomoto, N. Amemiya, X. Zhang and C. W. Bumby, "Dynamic resistance of a high-Tc coated conductor wire in a perpendicular magnetic field at 77K," Supercond. Sci. Technol., vol. 30, Jan. 2017, Art. no. 03LT01.

[24] G. P. Mikitik and E. H. Brandt, "Generation of a de voltage by an ac magnetic field in type II superconductors," Phsical Review B, vol. 64, Aug. 2001, Art. no. 92502.

[25] M. Iwakuma, M. Nigo, D. Inoue, N. Miyamoto, K. Funaki, Y. Iijima, T. Saitoh, Y. Yamada, T. Izumi, and Y. Shiohara, "AC loss properties of YBCO superconducting tapes fabricated by IBAD-PLD technique," Phys. C, vols. 412-414, pp. 983- 991, 2004.

[26] A. Palau, T. Puig, X. Obradors, E. Pardo, C. Navau, A. Sanchez, A. Usoskin, H. C. Freyhardt, L. Fernández, B. Holzapfel, and R. Feenstra, "Simultaneous inductive determination of grain and intergrain critical current densities of YBa2Cu3O7-x coated conductors," Appl. Phys. Lett, vol. 84, pp. 230-232, 2004.

[27] Z. Jiang, W. Zhou, Q. Li, M. Yao, J. Fang, N. Amemiya, and C. W. Bumby "The dynamic resistance of YBCO coated conductor wire: Effect of DC current magnitude and applied field orientation," Supercond. Sci. Technol., vol. 31, Jan. 2018, Art. no. 035002.

[28] S. C. Wimbush, and N. M. Strickland, "A Public Database of High-Temperature Superconductor Critical Current Data," IEEE Trans. Appl. Supercond, vol. 27, no. 4, pp. 1-5, June 2017.

[29] Y. Zhang, E. D. Specht, C. Cantoni, D. K. Christen, J. R. Thompson, J. W. Sinclair, A. Goyal, Y. L. Zuev, T. Aytug, M. P. Paranthaman, Y. Chen, and V. Selvamanickam. "Magnetic field orientation dependence of flux pinning in $(\mathrm{Gd}, \mathrm{Y}) \mathrm{Ba}_{2} \mathrm{Cu}_{3} \mathrm{O}_{7-\mathrm{x}}$, coated conductor with tilted lattice and nanostructures" Physica C, vol. 469, no. 23, pp. 2044-2051, August 2009. 